\title{
Picker Routing Problem: Taxonomia e métodos de resolução
}

Andrey A. H. Schulz (UFPR) a13_ahs@ hotmail.com

Cassius Tadeu Scarpin (UFPR) cassiusts@gmail.com

\section{Resumo}

A Indústria 4.0 traz consigo grandes expectativas, pois altera o modo como as empresas competem entre si. Esse conceito também se estende para a gestão da cadeia de suprimentos, o Supply Chain Management 4.0 (SCM 4.0), que se bem planejado possibilita vantagens competitivas. Assim, o foco torna-se a atividade de picking, a principal em um Centro de Distribuição. Surge então o Picker Routing Problem (PRP) que visa minimizar a distância total percorrida. O objetivo do trabalho é propor de uma taxonomia para o PRP e levantar seus métodos de resolução encontrados na literatura e baseados em técnicas de Pesquisa Operacional (PO). A contribuição deste trabalho reside no âmbito prático e teórico ao discorrer sobre o PRP sob a ótica da Indústria 4.0.

Palavras-Chaves: Indústria 4.0. Picker Routing Problem (PRP). Pesquisa Operacional

\section{Introdução}

O aumento do comércio global entre diferentes partes do mundo (GENG, LI e LIM, 2005) gerou uma acirrada concorrência (ENE E ÖZTÜRK, 2012) e as empresas se impulsionaram a desenvolver metodologias e processos mais completos para aumentar seu poder competitivo.

Diante disso, a aplicação de tecnologias disruptivas nas empresas está sendo caracterizada como uma nova revolução, também conhecida como manufatura inteligente ou Indústria 4.0 (FRAZZON et al., 2019). Os potenciais resultados dessa nova revolução trazem grandes expectativas, pois pode transformar o modo como as empresas competem entre si (PORTER \& HEPPELMANN, 2014).

Além disso, a necessidade de novos conceitos gerenciais capazes de refletir os desafios e oportunidade advindos da era digital, impulsionou as empresas também a repensarem o projeto de suas cadeias de suprimentos. Assim sendo, o conceito de Supply Chain Management (SCM) deveria se tornar Supply Chain Management 4.0 (SCM 4.0) (ALICKE et al., 2017; ASDECKER \& FELCH, 2018). 
Chopra e Meindl (2003) afirmam que para o cumprimento do alinhamento estratégico ao longo de toda cadeia de suprimento, uma empresa precisa estabelecer um equilíbrio entre responsividade e eficiência. Assim, para melhorar nesses dois aspectos, um dos fatores chave que se deve gerir é o Centro de Distribuição (CD) e suas atividades.

Destas, o picking (coleta de itens de um pedido) é considerada a atividade mais relevante por ser a mais dispendiosa. Grande parte dos estudos relacionados à essa atividade, sugere o roteamento dos pickers (separadores) como forma de potencializar a produtividade do $\mathrm{CD}$, pois objetiva encontrar uma distância total de percurso mínima, coletando todos os itens de um pedido (CHARKHGARD e SAVELSBERGH, 2015).

Assim, por meio de técnicas de otimização de Pesquisa Operacional (PO) torna-se possível quantificar e aperfeiçoar o picking de um CD de modo a diminuir os custos operacionais, proporcionar agilidade na execução da atividade e alavancar a produtividade da empresa (SCARPIN, 2012).

Nesse contexto, o objetivo deste trabalho, de caráter teórico e prático, é propor uma taxonomia para o problema de roteamento de pickers e levantar seus métodos de resolução encontrados na literatura e baseados em técnicas de PO.

\section{Referencial teórico}

A seguir, os principais conceitos relacionados a esse trabalho serão dissertados.

\subsection{Indústria 4.0}

O termo Indústria 4.0 foi inicialmente cunhado na Alemanha, em 2011. Desde então, academia e indústria apresentaram cada vez mais interesse nesse tema (FREDERICO et al., 2019). Naquela oportunidade, o conceito se baseava em quatro pilares: Soluções, Inovações, Cadeia de Suprimentos e Indústria Inteligentes (METHAVITAKUL \& SANTITEERAKUL, 2018).

Esse conceito também é chamado quarta revolução industrial (SILVA et al., 2018). A terminologia Revolução Industrial é aplicada quando existem mudanças significativas nos sistemas técnicos, econômicos ou social dentro da indústria, ou seja, uma mudança de paradigma no modelo da indústria (BIBBY \& DEHE, 2018). 
$\mathrm{Na}$ literatura, várias definições para Indústria 4.0 podem ser encontradas, a grande maioria aborda as tecnologias disponíveis e os impactos trazidos para dentro da empresa e/ou trazidos para a cadeia de valor. Assim sendo, pode-se dizer que a Indústria 4.0 é junção de todas as tecnologias disruptivas aplicadas em uma cadeia de suprimento com objetivo de integrar as tendências de digitalização, autonomização, transparência, mobilidade, modularização, colaboração em rede e socialização de produtos e processos (PFOHL et al., 2015).

No Quadro 1, são apresentados as tecnologias da Indústria 4.0:

Quadro 1 - Tecnologias da Indústria 4.0

\begin{tabular}{|c|c|}
\hline \multicolumn{2}{|r|}{ Tecnologias da Indústria 4.0} \\
\hline Dimensão & \begin{tabular}{|r|} 
Exemplo \\
\end{tabular} \\
\hline Análise de dados & Big Data e Mineração de Dados \\
\hline Distribuição de Dados & Computação na Nuvem \\
\hline $\begin{array}{c}\text { Visualização de Dados e } \\
\text { Cenários }\end{array}$ & Realidade Aumenada, simulação e realidade virtual \\
\hline Robotização Avançada & $\begin{array}{c}\text { Robôs autônomos, sistemas de automação, } \\
\text { inteligência artificial, Sistemas Cyber-Físicos, robôs } \\
\text { e veículos autônomos }\end{array}$ \\
\hline Sistemas Digitais/ Rede & $\begin{array}{c}\text { Internet das Coisas, Comunicação Máquina para } \\
\text { Máquina (M2M) e rede sem fio } \\
\end{array}$ \\
\hline $\begin{array}{c}\text { Sistemas de Segurança de } \\
\text { Dados }\end{array}$ & Segurança Cibernética e protocolo IPv6 \\
\hline $\begin{array}{c}\text { Coleta de Dados e } \\
\text { Controle de Equipamentos }\end{array}$ & $\begin{array}{c}\text { Aplicativos Móveis, sensores e Identificação por } \\
\text { Radio Frequência (RFID) }\end{array}$ \\
\hline Sistemas de Manufatura & $\begin{array}{c}\text { Manufatura Aditiva, Sistemas de integração } \\
\text { horizontal e vertical e impressão 3D }\end{array}$ \\
\hline
\end{tabular}

Fonte: Adaptado de Silva et al. (2018)

\subsection{Centro de distribuição (CD) e o picking}

O termo "armazém" é usado quando a função principal do estabelecimento é o armazenamento de materiais. Caso a função de distribuição também seja considerada, o termo “centro de distribuição" é comumente utilizado (KOSTER et al., 2007).

Estes estabelecimentos possuem como objetivo o equilíbrio e a acomodação da variabilidade de fornecimento gerada pela sazonalidade e/ou agrupamentos de materiais em lotes de produção ou lotes de transporte. Com isso, pode-se dizer que o CD representa um importante elo na cadeia de suprimentos ao armazenar e distribuir produtos nos pontos de origem, de 
consumo e/ou ao longo de toda cadeia (GU et al., 2007). As 4 atividades básicas de um CD são: recebimento, armazenamento, coleta das ordens (picking) e expedição (GILS et al, 2018). Do ponto de vista operacional, o picking consiste no percurso do picker no CD coletando os itens do estoque, requisitados por clientes, com uso de um dispositivo de transporte (carrinho) e transportando-os para o ponto de consolidação (depot) para embalagem e expedição (HALL, 1993).

Assim, fica evidente a influência do picking sobre o nível de serviço dos pedidos dos clientes (BURINSKIENE, 2010), ou seja, esta atividade representa um papel crucial na gestão da cadeia de suprimentos (DALLARI, et al., 2009). À vista disso, alguns autores estimaram que o custo do picking contempla entre 50\% (TOMPKINS et al, 2010) a até 75\% (COYLE et al, 1996) dos custos totais de um armazém.

Tompkins et al (2010) afirmam que o tempo total para realizar esta atividade pode ser dividido em diferentes tempos: para deslocamento (trajeto ou percurso), para busca do item, para coleta e para demais atividades (como descarregar os itens coletados). A distribuição dos tempos pode ser visto na Figura 1:

Figura 1 - Distribuição dos tempos do picking

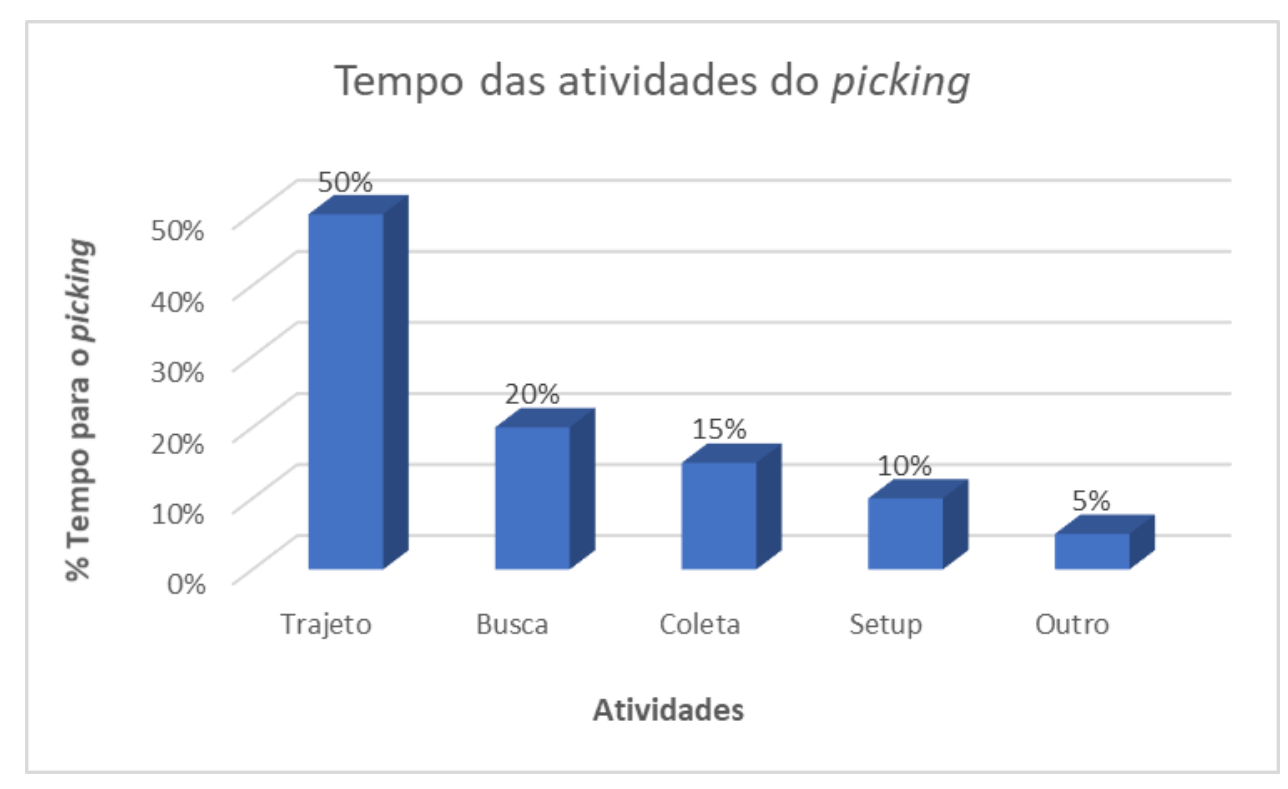

Fonte: Adaptado de Tompkins et al. (2010)

Assim o maior componente do tempo total do picking é o tempo gasto para deslocamento $(50 \%)$ até os pontos de coleta. Considera-se que esse tempo é improdutivo. 


\subsection{Roteamento em centro de distribuição}

Ao considerar que o tempo para o deslocamento, apresenta a maior parcela do picking, Bartholdi e Hackman (2005) enxergam esse tempo como um desperdício, visto que não agregar valor. Ademais, Koster et al. (2007) concluem que o tempo de deslocamento é o primeiro candidato para melhorias.

Assim sendo, como o tempo para o deslocamento é uma função crescente da distância a percorrer, uma maneira de potencializar a redução no tempo desta atividade é a minimização da distância a ser percorrida (SANTIS et al, 2018). Como consequência disso, a distância do percurso é com frequência considerada o objetivo principal em projetos e otimização de CDs (KOSTER et al,, 2007).

Na visão de Pesquisa Operacional, a partir da atividade do picking surge o problema de roteamento do picker, também chamado de Picking Routing Problem, que possui como objetivo a minimização da distância total percorrida no $\mathrm{CD}$, ao coletar todos os itens requisitados no pedido do cliente (SCHOLZ \& WÄSCHER, 2017). Em alguns casos, assume-se que o picker desloca-se em velocidade constante e assim, a minimização do percurso total equivale-se a minimização do tempo total do percurso (SCHOLZ et al., 2016).

\section{Metodologia}

Para a realização do estudo, a metodologia pôde ser dividida em duas etapas. Ambas as etapas trouxeram aspectos de uma revisão sistemática da literatura (RSL), porém, não abrangeram todo o rigor metodológico de uma RSL, ao invés disso, cada uma teve seu foco específico. De forma geral, esses foram os passos seguidos: a) seleção das bases de pesquisa; b) definição das palavras chaves; c) realização das buscas; d) seleção e qualificação dos artigos por meio da leitura de títulos e resumos; e) eliminação dos artigos duplicados; f) adição de novos artigos baseado nas referências de artigos qualificados.

A primeira etapa contemplou a pesquisa necessária para delinear quais são as tecnologias consideradas pela Indústria 4.0 e conseguir relaciona-las com o foco deste trabalho que é o Problema de Roteamento de Picker em um CD. As bases de pesquisa selecionadas foram: Web of Science, IEEE Xplore, Science Direct, Google Scholar. Como a gestão das atividades do CD é um assunto derivado de um tema maior, o Supply Chain, as palavras chaves foram: "Supply Chain 4.0". 
A segunda etapa buscou relacionar os achados da primeira etapa com os objetivos do trabalho de apresentar uma taxonomia para o roteamento do picker e levantar os métodos de resolução para o PRP baseados em técnicas de PO.

Para tanto, as bases de pesquisas selecionadas foram: Science Direct, Web of Science, Scopus, Google Scholar e IEEE Xplore. Os termos de pesquisa foram: "order picking" e "picking routing problem". Como a literatura retornou extensos resultados, os artigos selecionados foram aqueles que focavam única e exclusivamente no PRP.

\section{Desenvolvimento}

Após a leitura dos artigos qualificados, percebeu-se que existem vários tipos de CDs, com vários métodos de coleta e aspectos outros aspectos específicos.. Diante disso, propõe-se a Taxonomia básica para o Picker Routing Problem (PRP), conforme visto na Figura 2.

Figura 2 - Taxonomia para o PRP

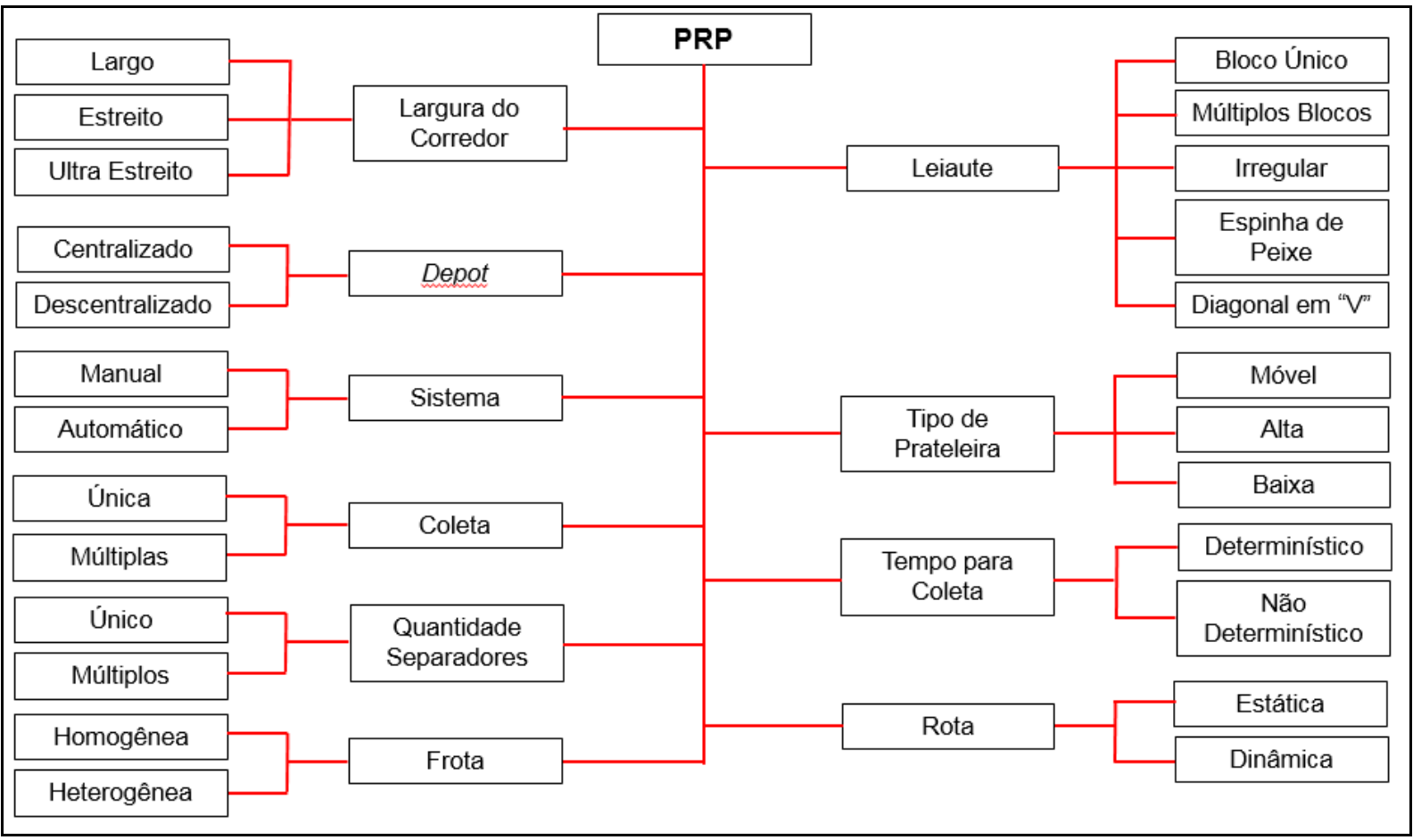

Fonte: O autor (2019)

Conforme a Figura 2, existem 10 aspectos a serem considerados, explicados no Quadro 2: 
Quadro 2 - Esclarecimento sobre dimensões da taxonomia

\begin{tabular}{|c|c|}
\hline \multicolumn{2}{|r|}{ Taxonomia } \\
\hline Dimensão & Esclarecimento \\
\hline Largura do Corredor & Espaço existe entre os corredores para a coleta dos itens \\
\hline Depot & $\begin{array}{l}\text { Local onde os pedidos coletados são entregues, podem ser em um só local ou } \\
\text { em vários }\end{array}$ \\
\hline Sistema & $\begin{array}{l}\text { Método de coleta dos itens a partir dos corredores onde os itens estão } \\
\text { armazenados }\end{array}$ \\
\hline Coleta & Quantos itens podem ser coletados uma vez que o carrinho do picker pára \\
\hline $\begin{array}{l}\text { Quantidade de } \\
\text { Separadores }\end{array}$ & Um ou mais separadores para cada pedido de um cliente individual \\
\hline Frota & $\begin{array}{l}\text { Tipo de carrinho considerado para armazenamento dos produtos que estão } \\
\text { sendo coletados }\end{array}$ \\
\hline Leiaute & Qual a disposição dos corredores de coleta dentro da estrutura física do galpão \\
\hline Tipo de Prateleira & Onde os itens a serem coletados estão armazenados \\
\hline Tempo para Coleta & Consideração de uma velocidade de deslocamento constante ou não \\
\hline Rota & $\begin{array}{l}\text { Leva em consideração outros acontecimentos no } \mathrm{CD} \text {, como presença de } \\
\text { outros pickers na mesma rota que poderia causar um congestionamento no } \\
\text { corredor, por exemplo }\end{array}$ \\
\hline
\end{tabular}

Fonte: O autor (2019)

Para cada uma das opções do PRP, pôde-se encontrar na literatura um sistema para otimização do problema. Esses sistemas se baseiam em técnicas de PO.

Vale citar que o trabalho de Frederico et al. (2019), que discorre sobre os conceitos e a maturidade do Supply Chain 4.0 (SC 4.0), a Revisão Sistemática da Literatura realizada tinha o objetivo de mapear as dimensões do SC 4.0. Dentre as dimensões citadas, os autores expandiram a dimensão de "Tecnologias Disruptivas" listando o que foi encontrado.

Um fato curioso, é que dos 24 artigos analisados, apenas um citava "Sistemas de Otimização" como uma tecnologia disruptiva para a Indústria ou Supply Chain 4.0. Acredita-se que isso se deve ao fato de que esta tecnologia já era utilizada antes do conceito de Indústria 4.0.

Considera-se isso verdadeiro, pois a área de PO existe desde o ano de 1934. O interesse por essa área aumentou durante a Segunda Guerra Mundial e desde então a área de PO com seus sistemas de otimização entraram no ambiente industrial (ARENALES et al., 2007).

Todavia, como a era digital gera enorme quantidade de dados a serem gerenciados e aproveitados (FREDERICO et al., 2019), os sistemas de otimização ganham novas perspectivas, ao surgirem novos campos de aplicação e modificações em aplicações já conhecidas devido a existência de novas informações. 
Diante disso, acredita-se que os Sistemas de Otimização passaram despercebidos quando as tecnologias da Indústria 4.0 foram citadas, no entanto, essa tecnologia deveria ser considerada com maior frequência. Fato similar acontece com os sensores, a simulação em tempo real, automação e robotização, que são utilizadas atualmente na indústria e que também são consideradas atrativas e perfeitas para a Indústria 4.0 (SILVA et al., 2018).

Dito isso, para o PRP encontrou-se os seguintes métodos de resolução: heurística, meta heurística e modelo exato. No Quadro 1, é apresentado a relação dos artigos e os tipos de resolução do PRP. Como algumas das heurísticas são bem difundidas e já foram bastante exploradas na literatura, estas possuem uma coluna específica. 
Quadro 3 - Relação entre artigos e métodos de solução para o PRP

\begin{tabular}{|c|c|c|c|c|c|c|c|c|c|c|c|c|}
\hline \multirow{2}{*}{ Autor(es) } & \multirow{2}{*}{ Ano } & \multicolumn{8}{|c|}{ Heurística } & \multirow{2}{*}{ Metaheuristica } & \multirow{2}{*}{$\begin{array}{l}\text { Modelo } \\
\text { Exato }\end{array}$} & \multirow{2}{*}{ Outros } \\
\hline & & S shape & return & midpoint & Combined & Combined+ & composite & largest gap & Aisle by Aisle & & & \\
\hline Koster et al. & 2007 & $x$ & $x$ & $x$ & $x$ & & & $x$ & & & $x$ & \\
\hline Gils et al. & 2018 & $x$ & $x$ & $x$ & & & & $x$ & $x$ & $x$ & $x$ & \\
\hline Chen et al. & 2018 & $x$ & $x$ & $x$ & & & & $x$ & & & & 6 novas heurísticas \\
\hline Scholz \& Wäscher & 2017 & $x$ & $x$ & $x$ & $x$ & $x$ & $x$ & $x$ & $x$ & & & \\
\hline Chabot et al. & 2016 & $x$ & & $x$ & $x$ & & & $x$ & & $x$ & $x$ & \\
\hline Roodbergen \& Koster & 2001 & $x$ & & & $x$ & $x$ & & $x$ & $x$ & & $x$ & \\
\hline Dukic \& Oluic & 2004 & $x$ & $x$ & $\mathrm{x}$ & $x$ & & & $x$ & & & $x$ & \\
\hline Hwang \& Lee & 2004 & $x$ & $x$ & $x$ & & & $x$ & $x$ & & & $x$ & \\
\hline Petersen II & 1999 & $x$ & & & & & $\mathrm{x}$ & $x$ & & & $x$ & \\
\hline Petersen II & 1997 & $x$ & $x$ & $x$ & & & $x$ & $\mathrm{x}$ & & & $x$ & \\
\hline Goetschalckx \& Ratliff & 1988 & $x$ & $x$ & & & & & & & & $x$ & \\
\hline Shouman et al. & 2001 & $x$ & & & $x$ & & & $x$ & $x$ & & & $\begin{array}{c}\text { Heurística Block } \\
\text { Aisle } 1 \text { e } 2\end{array}$ \\
\hline Charkhgard \& Savelsbergh & 2015 & $\mathrm{x}$ & $x$ & & & & $x$ & $x$ & & & & \\
\hline Burinskiene & 2010 & $x$ & $x$ & $x$ & $x$ & $x$ & $x$ & $x$ & $x$ & & $x$ & \\
\hline Hall & 1993 & $x$ & & $x$ & & & & $x$ & & $x$ & $x$ & \\
\hline
\end{tabular}

Fonte: O autor (2019) 
Assim sendo, as políticas de roteamento determinam as rotas a serem percorridas pelo picker (PETERSEN, 1999). Estas variam de simples heurísticas até algoritmos ótimos (SANTIS et al, 2018) passando também pelas meta-heurísticas. Assim, para a construção desses métodos de solução, ora o leiaute do CD é considerado ora não (ARDJMAND e HUH, 2017).

Os roteamentos ótimos, resultam em um menor tempo de percurso e tipicamente abordam o problema como um caso especial de Travelling Salesman Problem (TSP) (SANTIS et al, 2018) onde o layout é geralmente considerado, pois deve-se decidir se o método de resolução do TSP será simétrico ou assimétrico (LI, HUANG e DAI, 2016).

Apesar de retornar uma rota ótima, os modelos exatos apresentam pontos práticos negativos. Primeiramente, estes não estão disponíveis para todos os leiautes de CDs, pois, encontram-se apenas para configurações simples (SANTIS et al, 2018).

Segundo, as rotas geradas por esses algoritmos são geralmente complexas, ilógicas e de difícil entendimento aos separadores, que por consequência se desviam do percurso (GADEMANN e VELDE, 2005).

Logo, apesar de não resultar em um trajeto ótimo, as heurísticas são usadas na prática com mais frequência, pois se beneficiam das rotas geradas serem simples, de fácil entendimento e familiar aos separadores (PETERSEN, 1999).

Do ponto de vista dos métodos de solução, a principal dificuldade é que os modelos exatos não resolvem o problema em tempo hábil quando o tamanho do problema cresce. Por sua vez, as heurísticas e meta heurísticas nem sempre encontram a solução ótima (LI, HUANG e DAI, 2016).

Vale salientar que na literatura, os trabalhos comumente comparam a performance das heurísticas com modelos exatos (quando disponíveis) com o intuito de avaliar se os benefícios em termos de simplicidade e tempo computacional reduzido compensam o aumento no tempo de percurso gerado (SANTIS et al, 2018).

Mesmo com a extensa literatura a respeito de roteamento de separadores em CDs, aplicar abordagens gerais do problema em configurações de CD específicas, considerando projetos e políticas operacionais singulares tipicamente encontradas na prática, não é tão simples (ISLER et al., 2016). Consequentemente, vários trabalhos na literatura resolvem casos práticos específicos por meio de algoritmos "feitos sob medida". 
Além do mais, Cano et al. (2017) apresentam algumas tendências consideradas para o PRP, como:

- Incorporação de prazos nos pedidos de clientes devido a prioridades de cumprimento destes em determinado tempo;

- A criação de modelos multi-objetivos;

- Contextos dinâmicos (online) ao considerar a chegada contínua de pedidos e incorporação constante de novas informações no ambiente operacional;

- Múltiplos separadores;

- Investigação do impacto de diferentes leiautes na coleta de pedidos;

- Problema conjunto ao integrar coleta de pedidos com outras questões de planejamento como designação de produtos aos locais de armazenagem, agrupamento de pedidos, sequenciamento e roteamento.

\section{Conclusão}

Por meio dos vários artigos encontrados na literatura relacionados ao PRP, percebeu-se a riqueza de opções existentes para a atividade do picking. Diante disso, apresentou-se a Taxonomia para o problema. Em seguida, foi apresentado o que está sendo feito na literatura para a resolução do PRP.

Em contra partida, poucos artigos apresentaram os sistemas de otimização baseados em técnicas de PO, como tecnologias da Indústria 4.0. Dessa forma, vale a pena reforçar que os sistemas de otimização ganham um grande espaço na era de digitalização devido a abundância de dados gerados pelos elementos conectados na rede. É um campo que com grande potencial a ser explorado e que merece atenção dos pesquisadores e do setor industrial.

Além disso, a importância deste artigo reside tanto no âmbito teórico quanto prático. Na perspectiva teórica, a abordagem do trabalho relaciona a tecnologia de sistema de otimização com técnicas de PO como uma das alavancas tecnológicas da Indústria 4.0.

Na ótica prática, este artigo apresenta pontos a favor e contra aos métodos de resolução do PRP, suas características e uma breve tendência do que está sendo aplicado na área.

Ademais, acredita-se que alguns gaps da literatura podem ser encontrados após a leitura deste trabalho que podem ser abordados em trabalhos futuros, como o uso de sistemas de otimização em outras atividades do CD e principalmente a integração de outras tecnologias 
disruptivas (RFID, realidade aumentada, tecnologia dos sensores, robótica, simulação) da Indústria 4.0 no PRP.

Isso abre um leque para novos desenvolvimentos, pois com novas aplicações, existe a possibilidade de se considerar mais aspectos reais do problema. Por consequência, isso leva ao desenvolvimento de novas heurísticas, meta-heurística e modelos exatos para o PRP. Em paralelo a isso, existe o desafio de se elaborar, em um extremo, sistemas "feitos sob medida" e no outro, sistemas mais gerais para serem aplicados em várias das segmentações existentes do PRP.

\section{REFERÊNCIAS}

Alicke, K., REXBAUSEN,D. AND SEYFERT, A. (2017), Supply Chain 4.0 In Consumer Good, McKinsey and Company, Berlin, available at: www.mckinsey.com/industries/consumer-packaged-goods/ourinsights/supply-chain-4-0-in-consumer-goods

ARDJMAND, Ehsan; HUH, Dong Wook. Coordinated warehouse order picking and production scheduling: A NSGA-II approach. 2017 Ieee Symposium Series On Computational Intelligence (ssci), [s.1.], p.1-8, nov. 2017. IEEE. http://dx.doi.org/10.1109/ssci.2017.8280855.

ARENALES, M.; ARMENTANO, V.; MORABITO, R.; YANASSE, H. Pesquisa Operacional para Cursos de Engenharia. 2007.

ASDECKER, Björn; FELCH, Vanessa. Development of an Industry 4.0 maturity model for the delivery process in supply chains. Journal Of Modelling In Management, [s.1.], v. 13, n. 4, p.840-883, 5 nov. 2018. Emerald. http://dx.doi.org/10.1108/jm2-03-2018-0042.

BARTHOLDI, J.J., HACKMAN, S.T., 2005. Warehouse \& distribution science. Disponível em: https://www2.isye.gatech.edu/ spyros/courses/IE6202/Fall-2002/Bartholdi-Hackman.pdf

BIBBY, L; DEHE, B (2018). Defining and assessing industry 4.0 maturity levels-case of the defence sector. Production Planning and Control, 29 (12). pp. 1030-1043. ISSN 0953-7287

BURINSKIENE, A. Order picking process at warehouses, Int. J. Logistics Systems and Management, Vol. 6, No. 2, pp.162-178. 2010.

CANO, Jose A.; CORREA-ESPINAL, Alexander A.; GÓMEZ-MONTOYA, Rodrigo A.. A review of research trends in order batching, sequencing and picker routing problems. Espacios, [s.i], v. 39, n. 4, p.3-14, 2017.

CHABOT, Thomas et al. Order picking problems under weight, fragility and category constraints. International Journal Of Production Research, [s.1.], v. 55, n. 21, p.6361-6379, 27 out. 2016. Informa UK Limited. http://dx.doi.org/10.1080/00207543.2016.1251625

CHARKHGARD, H.; SAVELSBERGH, M.. Efficient algorithms for travelling salesman problems arising in warehouse order picking. The Anziam Journal, [s.1.], v. 57, n. 02, p.166-174, 22 set. 2015. Cambridge University Press (CUP). http://dx.doi.org/10.1017/s1446181115000140.

CHEN, Fangyu; XU, Gangyan; WEI, Yongchang. Heuristic routing methods in multiple-block warehouses with ultra-narrow aisles and access restriction. International Journal Of Production Research, [s.1.], v. 57, n. 1, p.228-249, 25 maio 2018. Informa UK Limited. http://dx.doi.org/10.1080/00207543.2018.1473657. 
CHEN, Tzu-li et al. An efficient hybrid algorithm for integrated order batching, sequencing and routing problem. International Journal Of Production Economics, [s.l.], v. 159, p.158-167, jan. 2015. Elsevier BV. http://dx.doi.org/10.1016/j.ijpe.2014.09.029.

CHENG, Chen-yang et al. Using a hybrid approach based on the particle swarm optimization and ant colony optimization to solve a joint order batching and picker routing problem. International Journal Of Production Economics, [s.1.], v. 170, p.805-814, dez. 2015. Elsevier BV. http://dx.doi.org/10.1016/j.ijpe.2015.03.021.

CHOPRA, Sunil; MEINDL, Peter. Gerenciamento da Cadeia de Suprimentos: Estratégia, Planejamento e Operações. São Paulo: Pearson Prentice Hall, 2003. 465 p.

COYLE, J.J., BARDI, E.J., LANGLEY, C.J., 1996. The Management of Business Logistics, 6th Edition. West Publishing, St. Paul, MN.

DALLARI, Fabrizio; MARCHET, Gino; MELACINI, Marco. Design of order picking system. The International Journal Of Advanced Manufacturing Technology, [s.1.], v. 42, n. 1-2, p.1-12, 17 jun. 2008. Springer Nature. http://dx.doi.org/10.1007/s00170-008-1571-9.

DUKIC, Goran; Oluic, Cedomir. Order-Picking Routing Policies: Simple Heuristics, Advanced Heuristics or Optimal Algorithm. Journal of Mechanical Engineering. P.530-535. ISSN: 0039-2480.

ENE, Seval; ÖZTÜRK, Nursel. Storage location assignment and order picking optimization in the automotive industry. The International Journal Of Advanced Manufacturing Technology, [s.1.], v. 60, n. 5-8, p.787-797, 30 ago. 2011. Springer Nature. http://dx.doi.org/10.1007/s00170-011-3593-y.

FRAZZON, Enzo Morosini et al. Towards Supply Chain Management 4.0. Brazilian Journal Of Operations \& Production Management, [s.1.], v. 16, n. 2, p.180-191, 25 maio 2019. Associacao Brasileira de Engenharia de Producao - ABEPRO. http://dx.doi.org/10.14488/bjopm.2019.v16.n2.a2.

FREDERICO, Guilherme F. et al. Supply Chain 4.0: concepts, maturity and research agenda. Supply Chain Management: An International Journal, [s.l.], v. --, n. --, p.1-21, 14 set. 2019. Emerald. http://dx.doi.org/10.1108/scm-09-2018-0339.

GADEMANN, Noud; VELDE, Steef. Order batching to minimize total travel time in a parallel-aisle warehouse. Iie Transactions, [s.1.], v. 37, n. 1, p.63-75, jan. 2005. Informa UK Limited. http://dx.doi.org/10.1080/07408170590516917.

GENG, Yue; LI, Yanzhi; LIM, A.. A very large-scale neighborhood search approach to capacitated warehouse routing problem. 17th Ieee International Conference On Tools With Artificial Intelligence (ictai'05), [s.l.], p.3-10, 2005. IEEE. http://dx.doi.org/10.1109/ictai.2005.21.

GILS, Teun Van et al. Increasing order picking efficiency by integrating storage, batching, zone picking, and routing policy decisions. International Journal Of Production Economics, [s.1.], v. 197, p.243-261, mar. 2018. Elsevier BV. http://dx.doi.org/10.1016/j.ijpe.2017.11.021.

GOETSCHALCKX, Marc; RATLIFF, H. Donald. Order Picking In An Aisle. Iie Transactions, [s.1.], v. 20, n. 1, p.53-62, mar. 1988. Informa UK Limited. http://dx.doi.org/10.1080/07408178808966150.

GU, Jinxiang; GOETSCHALCKX, Marc; MCGINNIS, Leon F.. Research on warehouse operation: A comprehensive review. European Journal Of Operational Research, [s.1.], v. 177, n. 1, p.1-21, fev. 2007. Elsevier BV. http://dx.doi.org/10.1016/j.ejor.2006.02.025.

HALL, Randolph W.. DISTANCE APPROXIMATIONS FOR ROUTING MANUAL PICKERS IN A WAREHOUSE. Iie Transactions, [s.1.], v. 25, n. 4, p.76-87, jul. 1993. Informa UK Limited. http://dx.doi.org/10.1080/07408179308964306.

HWANG, H.; OH, Y. H.; LEE, Y. K.. An evaluation of routing policies for order-picking operations in low-level picker-to-part system. International Journal Of Production Research, [s.l.], v. 42, n. 18, p.3873-3889, 15 set. 2004. Informa UK Limited. http://dx.doi.org/10.1080/00207540410001696339. 
ISLER, C. A.; RIGHETTO, G. M.; MORABITO, R.. Optimizing the order picking of a scholar and office supplies warehouse. The International Journal Of Advanced Manufacturing Technology, [s.1.], v. 87, n. 5-8, p.2327-2336, 23 mar. 2016. Springer Nature. http://dx.doi.org/10.1007/s00170-016-8625-1.

KOSTER, René de; LE-DUC, Tho; ROODBERGEN, Kees Jan. Design and control of warehouse order picking: A literature review. European Journal Of Operational Research, [s.1.], v. 182, n. 2, p.481-501, out. 2007. Elsevier BV. http://dx.doi.org/10.1016/j.ejor.2006.07.009.

LI, Jianbin; HUANG, Rihuan; DAI, James B.. Joint optimisation of order batching and picker routing in the online retailer's warehouse in China. International Journal Of Production Research, [s.1.], v. 55, n. 2 , p.447461, 19 maio 2016. Informa UK Limited. http://dx.doi.org/10.1080/00207543.2016.1187313.

METHAVITAKUL, Banthia; SANTITEERAKUL, Salinee. Analysis of key dimension and sub-dimension for Supply Chain of Industry to fourth Industry Performance Measurement. International Conference on Service Operations and Logistics and Informatics (SOLI), p. 191-195, 2018.

PETERSEN, Charles G.. An evaluation of order picking routeing policies. International Journal Of Operations \& Production Management, [s.1.], v. 17, n. 11, p.1098-1111, nov. 1997. Emerald. http://dx.doi.org/10.1108/01443579710177860.

PETERSEN, Charles G.. The impact of routing and storage policies on warehouse efficiency. International Journal Of Operations \& Production Management, [s.1.], v. 19, n. 10, p.1053-1064, out. 1999. Emerald. http://dx.doi.org/10.1108/01443579910287073.

PFOHL, H., YAHSI, B. and KURNAZ, T. (2015), The impact of industry 4.0 on supply chain. Proceedings of the Hamburg International Conference of Logistics. Hamburg, Germany, 2015, available at: https://hicl.org/publications/2015/20/31.pdf

PORTER, M.E.; HEPPELMANN, J.E. (2014), "How smart, connected products are transforming competition", Harvard Business Review, Vol. 92 No. 11, pp. 64-88, disponível em: https://hbr.org/2014/11/how-smartconnected-products-aretransforming-competition

ROODBERGEN, Kees Jan; KOSTER, René de. Routing methods for warehouses with multiple cross aisles. International Journal Of Production Research, [s.1.], v. 39, n. 9, p.1865-1883, jan. 2001. Informa UK Limited. http://dx.doi.org/10.1080/00207540110028128.

SANTIS, Roberta de et al. An adapted ant colony optimization algorithm for the minimization of the travel distance of pickers in manual warehouses. European Journal Of Operational Research, [s.1.], v. 267, n. 1, p.120-137, maio 2018. Elsevier BV. http://dx.doi.org/10.1016/j.ejor.2017.11.017.

SCARPIN, C. T. Uma metodologia para a previsão de demanda de produtos utilizando redes neurais artificiais de funções de bases radiais modificadas e uma proposta de logística de reposição, 2012. UFPR.

SCHOLZ, André et al. A new mathematical programming formulation for the Single-Picker Routing Problem. European Journal Of Operational Research, [s.1.], v. 253, n. 1, p.68-84, ago. 2016. Elsevier BV. http://dx.doi.org/10.1016/j.ejor.2016.02.018.

SCHOLZ, A.; WÄSCHER, G.. Order Batching and Picker Routing in manual order picking systems: the benefits of integrated routing. Central European Journal Of Operations Research, [s.1.], v. 25, n. 2, p.491-520, 31 jan. 2017. Springer Nature. http://dx.doi.org/10.1007/s10100-017-0467-x.

SHOUMAN, M. A. et al. Comparisons of order picking routing methods for warehouses with multiple cross aisles. 2001

SILVA, Vander Luiz da; KOVALESKI, João Luiz; PAGANI, Regina Negri. Technology transfer in the supply chain oriented to industry 4.0: a literature review. Technology Analysis \& Strategic Management, [s.1.], v. 31, n. 5, p.546-562, 22 set. 2018. Informa UK Limited. http://dx.doi.org/10.1080/09537325.2018.1524135.

TOMPKINS, et al. 2010. Facilities Planning. John Wiley \& Sons, Hoboken, NJ. 